AIChE Journal, 2009, 55(10), 2553-2562, doi : 10.1002/aic.11870

\title{
Precipitation dynamics of zinc sulfide multi-scale agglomerates
}

\author{
F. GRUY, M.K. MEKKI-BERRADA, M. COURNIL ${ }^{*}$ ) \\ Département GENERIC, Centre SPIN, Ecole Nationale Supérieure des Mines de Saint- \\ Etienne, LPMG UMR 5148, 158 Cours Fauriel 42023 Saint-Etienne, France \\ (*) cournil@emse.fr
}

\section{ABSTRACT}

Homogeneous precipitation of zinc sulfide from thioacetamide decomposition in presence of zinc sulfate in acid solution results in the formation of four-scale agglomerates. We present here experimental results relative to the monitoring of several physicochemical $(\mathrm{pH}$, electrical conductivity, concentration in sulfide ions, turbidity) and morphological (agglomerate shape and size) parameters throughout the precipitation process. From these parameters essential characteristics of the precipitation process can be determined, especially the supersaturation level and the precipitated product mass. From this information and with the help of microphotographs of samples withdrawn at different stages of the precipitation, it is possible to formulate a new mechanism of zinc sulfide precipitation. In particular the nature of the different agglomeration scales is elucidated as well their succession in time.

Keywords:

precipitation ; agglomeration ; zinc sulfide ; multi-scale agglomerates

Topical area:

particle technology and fluidization

\section{Introduction:}

In precipitation processes final product is often obtained in the form of agglomerates of particles, which themselves consist of assembling of smaller crystals. This structure is sometimes observed over several successive scales [1-11]. Clusters with such morphology are called multi-scale agglomerates. They offer several advantages for industrial applications because according to the scale under consideration different functions can be solicited, i.e. mechanical, catalytic, reactive, and so on. Available literature about multi-scale agglomeration is mainly experimental and concerns zinc sulfide precipitation in most cases [1-9].

Zinc sulfide $(\mathrm{ZnS})$ precipitates are known indeed to present four scales of agglomeration particularly when they are obtained from homogeneous precipitation. Thermal decomposition of thioacetamide $\left(\mathrm{C}_{2} \mathrm{H}_{5} \mathrm{NS}\right)$, (TAA) in an acid solution of zinc sulfate creates the supersaturation conditions necessary to $\mathrm{ZnS}$ homogeneous precipitation. The mechanism proposed by Eshuis et al. [8-9] consists of different steps of nucleation-growth and successive agglomerations (Figure 1) which are not all incontestably identified nor justified.

With other products, David et al [10] and Cameirão [11] proved the respective influence of Brownian and laminar agglomeration on the observed scale sizes.

In a general way, origin of multi-scale morphology is not always clearly understood, in particular for lack of sufficient knowledge of time evolution of the system throughout the precipitation process.

One of us recently performed to this aim a quantitative study of zinc sulfide homogeneous precipitation [12]. We present here a set of results drawn from his work and elements of modeling which should allow to progress in the comprehension of the dynamics of formation of multi-scale agglomerates.

This paper is structured as follows. Next section deals with materials and methods. Zinc sulfide homogeneous precipitation process is described as well as the experimental set-up, protocol and techniques that are used in this work. Exhaustive exploitation of some in-line measurements requires both theoretical and experimental study which is presented then. 
AIChE Journal, 2009, 55(10), 2553-2562, doi : 10.1002/aic.11870

Afterwards dynamics of $\mathrm{ZnS}$ precipitation itself is determined using different experimental results and calculated data. Lastly a new mechanism of precipitation of the multi-scale agglomerates is proposed and discussed.

\section{Materials and Methods}

\section{Chemical Aspects}

The homogeneous precipitation principle is to slowly release one of the reactants in situ so that a moderate however particularly uniform supersaturation level is reached which is generally favorable for obtaining narrow and uniform particle size distribution [6]. In case of $\mathrm{ZnS}$ precipitation, initial solution is a homogeneous mixture of $\mathrm{Zn}^{2+}$ ions and thioacetamide molecules.

Thioacetamide (TAA) decomposition is an irreversible reaction which is catalyzed by $\mathrm{H}_{3} \mathrm{O}^{+}$ ions and proceeds with a noticeable rate above $60^{\circ} \mathrm{C}\left[13^{-15}\right]$.

$$
\mathrm{CH}_{3} \mathrm{CSNH}_{2}+\mathrm{H}_{2} \mathrm{O} \rightarrow \mathrm{CH}_{3} \mathrm{CONH}_{2}+\mathrm{H}_{2} \mathrm{~S}
$$

Sulfide ions coming from the dissociation of so produced hydrogen sulfide react with zinc ions to give zinc sulfide precipitate.

$$
\mathrm{Zn}^{2+}{ }_{(\mathrm{aq})}+\mathrm{S}^{2-}{ }_{(\mathrm{aq})} \rightarrow \mathrm{ZnS}_{(\mathrm{s})}
$$

Thioacetamide decomposition proceeds in fact along two parallel paths $\left(R_{1}-R_{3}\right.$ and $\left.R_{2}-R_{4}\right)$ whose kinetics is relatively well known [13-15]. In this work we use the Peeters and de Ranter model [14] which is stated in the following way:

$$
\begin{array}{ll}
\mathrm{CH}_{3} \mathrm{CSNH}_{2}+\mathrm{H}_{2} \mathrm{O} \rightarrow \mathrm{CH}_{3} \mathrm{CONH}_{2}+\mathrm{H}_{2} \mathrm{~S} & \left(R_{1} \text { : rate constant } k_{1}\right) \\
\mathrm{CH}_{3} \mathrm{CONH}_{2}+\mathrm{H}_{3} \mathrm{O}^{+} \rightarrow \mathrm{CH}_{3} \mathrm{CO}_{2} \mathrm{H}+\mathrm{NH}_{4}^{+} & \left(R_{3} \text { : rate constant } k_{3}\right) \\
\mathrm{CH}_{3} \mathrm{CSN} \mathrm{H}_{2}+\mathrm{H}_{3} \mathrm{O}^{+} \rightarrow \mathrm{CH}_{3} \mathrm{CSOH}+\mathrm{NH}_{4}^{+} & \left(R_{2} \text { : rate constant } k_{2}\right) \\
\mathrm{CH}_{3} \mathrm{CSOH}+\mathrm{H}_{2} \mathrm{O} \rightarrow \mathrm{CH}_{3} \mathrm{CO}_{2} \mathrm{H}+\mathrm{H}_{2} \mathrm{~S} & \left(R_{4} \text { : rate constant } k_{4}\right)
\end{array}
$$

\section{Experimental Set-Up}

Experimental set-up (Figure 2) consists of a double-jacketed 2.5 L cylindrical main reactor and an auxiliary $1.5 \mathrm{~L}$ reactor. Precipitation takes place in the main reactor which is fitted with four Teflon baffles and stirred with a $45^{\circ}$ four-blade propeller stirring rate $N$. Excess of gaseous hydrogen sulfide is entrained by a low nitrogen flow-rate $(1 \mathrm{~N} \mathrm{~L} /$ hour $)$ to the auxiliary reactor in which it is absorbed by a $0.5 \mathrm{M}$ soda aqueous solution.

\section{Experimental Protocol}

Zinc sulfide $\left(\mathrm{ZnSO}_{4} \cdot 7 \mathrm{H}_{2} \mathrm{O}\right)$ and thioacetamide are Aldrich products of $99 \%$ purity. Reactants are initially introduced at ambient temperature into the main reactor: thioacetamide (variable concentration), zinc sulfate (constant concentration: $0.05 \mathrm{M}$ ) and nitric acid (to adjust the $\mathrm{pH}$ at the desired value). Reactor is thermostated at $25^{\circ} \mathrm{C}$. In these conditions, initial mixture is inert. To obtain sufficiently high and rapid decomposition of TAA, heating up to working temperature (higher than $60^{\circ} \mathrm{C}$ ) is operated and then maintained at constant value for about 3 hours and finally decreased to ambient temperature by natural cooling as described in the diagram of Figure 3. Precipitation is liable to proceed as soon as sulfide ions are released, thus in the follow-up, the instant when temperature exceeds $60^{\circ} \mathrm{C}$ will be taken as zero time of the precipitation process (after about 40 minutes of heating). For off-line characterization, zinc sulfide suspension samples are collected, withdrawn and filtered at $0.45 \mu \mathrm{m}$ then washed five times with distillated water and lastly dried at $50^{\circ} \mathrm{C}$ for 12 hours. It is checked that these treatments do not damage the collected powder.

\section{Characterization Methods}

Dynamics of $\mathrm{ZnS}$ precipitation will be interpreted from observations or measurements operated in a discrete or continuous way at different times of the process. 
AIChE Journal, 2009, 55(10), 2553-2562, doi : 10.1002/aic.11870

Off-line methods

* Gravimetry : mass of precipitated $\mathrm{ZnS}$ is determined by weighing of the removed samples;

* Particle size distribution is measured by laser beam diffraction in a Malvern 2000 apparatus;

* The morphology of the agglomerates is examined by scanning electronic microscopy (SEM) (JEOL JSM-6500 FEG-SEM); some scale sizes are directly measured from these images.

In situ methods

* $\mathrm{pH}$ is determined from the electromotive force delivered by a $\mathrm{pH}$ combination electrode;

* Electrical conductivity is continuously recorded in the two reactors;

* From polychromatic turbidity signal measured in main reactor, it is possible to get information on $\mathrm{ZnS}$ particle size distribution at early stage of the precipitation and more generally as long as submicronic particles are present in the medium [16];

* Concentration in sulfide ions in auxiliary reactor is determined with an ionic specific electrode ELIT.

\section{Experimental Results}

\section{Global characterization of the precipitation process}

In this section morphological aspects of the $\mathrm{ZnS}$ agglomerates are not considered. Particular interest is given to mass transfer between the two reactors and between liquid and solid phases. We explain first how the previous measurements can be exploited to characterize the liquid solutions in each reactor and then to calculate essential parameters especially the mass of precipitated zinc sulfide and supersaturation level in main reactor. For this determination we need to compare TAA decomposition in presence and in absence $\mathrm{ZnSO}_{4}$. The experiments which are commented on in the sequel were performed in the following experimental conditions: $[\mathrm{TAA}]=0.1 \mathrm{~mol} . \mathrm{L}^{-1},\left[\mathrm{ZnSO}_{4}\right]=0.025 \mathrm{~mol} . \mathrm{L}^{-1}, \mathrm{pH}=1.5$, $T_{\mathrm{R} 1}=80^{\circ} \mathrm{C} ; N=700 \mathrm{rpm}$.

TAA decomposition in absence of $\mathrm{ZnSO}_{4}$

The experimental set-up and protocols are the same as those described in section 2; operating conditions are specified just above except that the initial medium does not contains zinc sulfate.

Figures $4 \mathrm{a}, 4 \mathrm{~b}$ respectively show the electrical conductivity and $\mathrm{pH}$ electrode potential variation with time in main reactor. As expected, conductivity starts by increasing due to the temperature effect; then it decreases because reactions $R_{2}$ and $R_{3}$ occur indeed and $\mathrm{H}_{3} \mathrm{O}^{+}$ions are gradually replaced by less mobile $\mathrm{NH}_{4}{ }^{+}$, whereas $\mathrm{pH}$ slightly increases. Produced $\mathrm{H}_{2} \mathrm{~S}$ remains partially in the solution, however is found also in the gas sky of main reactor and is absorbed in auxiliary reactor. Determination of total sulfur in the auxiliary reactor can be performed from measurements of conductivity or concentration in sulfide ion measurements. This determination requires the knowledge of $\mathrm{HS}^{-} / \mathrm{S}^{2-}$ couple acidity constant and of the equivalent ionic conductivities [12] (see Appendix A).

Figure 5 shows the variation of total sulfur concentration $\left[S_{T}\right]_{R 2}$ with time in the auxiliary reactor.

Plots are quite similar whether they are calculated from conductivity (solid line) or from specific electrode (dotted line) measurements. Main interest of these plots is that their slope is equal to the flow-rate of absorbed $\mathrm{H}_{2} \mathrm{~S}$. This parameter will be used in the calculation of total sulfur and other species concentration in main reactor. Calculation steps are as follows: 
AIChE Journal, 2009, 55(10), 2553-2562, doi : 10.1002/aic.11870

the $\mathrm{H}_{2} \mathrm{~S}$ mole flow-rate transferred to auxiliary reactor via the gas phase is given by:

$$
{\stackrel{R}{n_{H_{2} S}}}^{R 2}=\frac{P_{H_{2} S}}{P_{T}} \dot{n}_{T}
$$

where $n_{T}$ is the total mole flow-rate of the gas phase, $P_{\mathrm{T}}$ and $P_{H_{2} S}$ respectively the $\mathrm{H}_{2} \mathrm{~S}$ partial pressure and the total pressure.

Hence:

$$
\dot{n}_{H_{2} S}^{R 2}=\frac{P_{H_{2} S}}{P_{T}-P_{H_{2} S}} \dot{n}_{N_{2}}
$$

$\dot{n}_{N_{2}}$, the mole flow-rate of nitrogen can be deduced from known volume flow-rate $\dot{V}_{N_{2}}$ at the gas inlet temperature $T_{N_{2}}$ :

$$
\dot{n}_{N_{2}}=\frac{P_{T} \dot{V}_{N_{2}}}{R T_{N_{2}}}
$$

According to Henry's law, $\mathrm{H}_{2} \mathrm{~S}$ partial pressure is linked to $\mathrm{H}_{2} \mathrm{~S}$ concentration in main reactor $C_{H_{2} S}^{R 1}$ by relation:

$$
C_{H_{2} S}^{R 1}=K_{H} P_{H_{2} S}
$$

Where $K_{\mathrm{H}}$ is the Henry constant $\left(K_{\mathrm{H}}=4 \times 10^{-7} \mathrm{~mol} \cdot \mathrm{L}^{-1} \cdot \mathrm{Pa}^{-1}\right.$ at $\left.80^{\circ} \mathrm{C}\right)$.

From Equations (2-4), we derive:

$$
C_{H_{2} S}^{R 1}=K_{H} P_{T} \frac{A}{1+A}
$$

With $A=\dot{n}_{H_{2} S}^{R 2} \frac{R T_{N_{2}}}{P_{T} \dot{V}_{N_{2}}}$

Operating factors $T_{N_{2}}, \dot{V}_{N_{2}}, P_{T}$ are fixed and $\dot{n}_{H_{2} S}$ is obtained from the experiments (slope of plots in Figure 5), $A$ and thus $C_{H_{2} S}^{R 1}$ can be evaluated using (5) and (6).

Hence the total sulfur concentration $\left[S_{T}\right]_{R 1}$ in the system, expressed relatively to the main reactor volume $V_{\mathrm{R} 1}$, is given by:

$$
\left[S_{T}\right]_{R 1}=C_{H_{2} S}^{R 1}+\frac{P_{H_{2} S}}{R T_{R 1}} \frac{V_{N_{2}}}{V_{R 1}}+\left[S_{T}\right]_{R 2}
$$

$V_{N_{2}}$ and $T_{\mathrm{R} 1}$ are respectively the volume of the reactor gas sky and the temperature in main reactor.

\section{Remarks:}

The remarks are as follows :

i) considering the imposed nitrogen flow-rate of $1.2 \mathrm{NL} / \mathrm{min}$, as maximum $\mathrm{H}_{2} \mathrm{~S}$ partial pressure is the atmospheric pressure, corresponding mole flow-rate entering auxiliary reactor is $0.00033 \mathrm{~mol} . \mathrm{L}^{-1}$. $\mathrm{min}^{-1}$. At the present $\mathrm{pH}$ value of 1.5 , the $\mathrm{H}_{2} \mathrm{~S}$ flow-rate 
AIChE Journal, 2009, 55(10), 2553-2562, doi : 10.1002/aic.11870

calculated from Figure 5 is actually lower than this limit. This means that nitrogen flowrate imposes indeed $\mathrm{H}_{2} \mathrm{~S}$ transfer between the two reactors.

ii) using the Peeters and de Ranter model [14], it is also possible to determine the total sulfur concentration $\left[S_{T}\right]_{R 1}$ from kinetic calculation [12] (see Appendix B). Figure 6 shows the variation of $\left[S_{T}\right]_{R 1}$ with time (experimental and theoretical determination). The relatively good agreement between the two methods of determination can be considered as validation of each of them and particularly of the experimental method which we can apply now to the complete system: TAA, $\mathrm{ZnSO}_{4}, \mathrm{HNO}_{3}$. The origin of the increasing deviation between the experimental values and the model at times beyond 100 minutes is not quite clear. It is much lower at other $\mathrm{pH}$ values (0.5 and 2.5), however is also observed for calculated and measured $\mathrm{NH}_{4}{ }^{+}$concentrations. We suspect in fact a modeling problem with Peeters and de Ranter kinetic scheme. This discrepancy, however, is not really embarrassing, because, as seen later on in this paper, the essential information is the comparison between $\left[S_{T}\right]_{R 1}$ calculated and measured in presence or in absence of $\mathrm{ZnSO}_{4}$ rather than each of these values.

TAA decomposition in presence of $\mathrm{ZnSO}_{4}$

Initial conditions are again $[\mathrm{TAA}]=0.1 \mathrm{~mol} . \mathrm{L}^{-1}, \mathrm{pH}=1.5, T_{\mathrm{R} 1}=80^{\circ} \mathrm{C} ; N=700 \mathrm{rpm}$ and $\left[\mathrm{ZnSO}_{4}\right]=0.025 \mathrm{~mol}^{-\mathrm{L}^{-1}}$. This means that zinc sulfide precipitation can occur now. We have examined its consequences on the same parameters as previously using the same determination procedures. For lack of space we only present here the variation of total sulfur concentration with time in main reactor $\left[S_{T}\right]_{\mathrm{TAA}_{\mathrm{ZnSO}}}$ in Figure 7.

In the same figure we have reported the calculated total sulfur concentration in case of [TAA] decomposition only $\left[S_{T}\right]_{\text {TAA }}$ (however taking into account the actual variation in $\mathrm{pH}$ for the kinetics calculation). Difference between the respective plots is due to the consumption of $\mathrm{H}_{2} \mathrm{~S}$ by zinc ions. Amount of precipitated $\mathrm{ZnS}$ is obtained by subtraction of previous quantities, that is to say: $[\mathrm{ZnS}]_{\mathrm{R} 1}=\left[S_{T}\right]_{\mathrm{TAA}}-\left[S_{T}\right]_{\mathrm{TAA}+\mathrm{ZnSO}_{4}}$

$[\mathrm{ZnS}]_{\mathrm{R} 1}$ denotes the quantity of precipitated zinc sulfide per reactor liter. Figure 8 shows the precipitation yield $[\mathrm{ZnS}]_{\mathrm{R} 1} /[\mathrm{ZnS}]_{\max }$ as a function of time. $[\mathrm{ZnS}]_{\max }$, maximum value of $[\mathrm{ZnS}]_{\mathrm{R} 1}$ is equal to initial concentration in $\left[\mathrm{Zn}^{2+}\right]$, i.e., $0.025 \mathrm{~mol} . \mathrm{L}^{-1}$. Precipitation is detected at the latest after 50 minutes of heating or as aforesaid ten minutes after the supersaturation onset. It is clear that precipitation is not completed at the end of present experiments and that precipitated mass is proportional to time. These results are widely confirmed by weighing samples removed from the solution. Due to the difficulty of this operation and the global reliability of our calculation procedure based on mostly experimental results, the latter will be preferred in the follow-up.

Concentration in dissolved $\left[\mathrm{Zn}^{2+}\right]$ is the difference between initial concentration in zinc ions and $[\mathrm{ZnS}]_{\mathrm{R} 1}$. Concentration in unreacted sulfide ions is obtained starting from total sulfur concentration (given by Eqn [7]) and requires relatively elementary calculation of acid-base equilibria in solution. It is necessary however to take into account $\mathrm{pH}$ variation throughout the precipitation process [12]. Supersaturation level $S$ is defined by:

$$
S=\sqrt{\frac{\left[\mathrm{Zn}^{2+}\right]\left[\mathrm{S}^{2-}\right]}{K_{s p}}}
$$

Where $K_{\text {sp }}$ denotes ZnS solubility product.

Variation of supersaturation with time is reported in Figure 9. Time zero is taken at the theoretical beginning of the precipitation process $\left(\mathrm{T}=60^{\circ} \mathrm{C}\right)$. 
AIChE Journal, 2009, 55(10), 2553-2562, doi : 10.1002/aic.11870

Supersaturation increases first because $\mathrm{H}_{2} \mathrm{~S}$ production by TAA decomposition is higher than consumption by $\mathrm{ZnS}$ nucleation and growth. Further supersaturation decrease is relatively slow.

\section{Agglomerate Morphology}

General morphological features of the agglomerates

Figure 10a shows a general view of the $\mathrm{ZnS}$ particles when removed at the end of the experiment. Figures $10 \mathrm{~b}$ to $10 \mathrm{~d}$ clearly highlight the successive scales of the agglomerates. Scale 1 corresponds to 5 to $40 \mathrm{~nm}$ crystallites. These crystallites seem to be grouped themselves in oval structures in form of "caterpillars" of about 150 to $400 \mathrm{~nm}$ length (scale 2). Scale 3 corresponds to spheres 1 to $6 \mu \mathrm{m}$ which are themselves gathered in relatively ramified agglomerates of 6 to $50 \mu \mathrm{m}$ (scale 4). This succession of structures is observed practically for all the experiments which are described here.

Variation of the agglomerate morphological characteristics during precipitation

Zinc sulfide precipitation is supposed to start as soon as a sufficient supersaturation level is reached, i.e., a few minutes after the beginning of TAA decomposition. For the thermal protocol which is adopted here, this means after 40 minutes heating. Previous photographs (Figure 10) are relative to "final" agglomerates, i.e. to samples withdrawn from the solution after three hours of experiments or more. However, to propose a mechanism for the precipitation process especially as regards its morphological aspects, observation of $\mathrm{ZnS}$ samples removed at earlier times from the suspension is particularly useful. In Figure 11, we present microphotographs taken at the same magnification of $\mathrm{ZnS}$ samples removed at different times. Time zero corresponds to the beginning of TAA decomposition (onset of supersaturation).

It clearly appears on these pictures that:

* multi-scale structure appears relatively early ; in particular, isolated spheres (scale 3 ) is observed as early as 30 minutes;

* scale 2 (caterpillars) is never observed separately but seems to be structured on the spot;

* development of large agglomerates seems to be globally homothetic.

From these microphotographs and others, we can derive the variation with time of the third scale size of the agglomerates (sphere diameter) as shown in Figure 12. For each point presented in Figure 12 about a hundred agglomerates were taken into account.

Linear variation is observed as early as the end of the nucleation step.

Particle sizing during precipitation

Two types of measurements were performed.

The first one, by laser diffraction on samples withdrawn at different precipitation times, provides results presented in Figure 13.

A peak is detected in the size distribution at about $0.5 \mu \mathrm{m}$ as far as 35 minutes and disappears then. From the beginning on a sharp distribution maximum is observed around 10 $\mu \mathrm{m}$. With the time it shifts to larger sizes however its intensity does not considerably change. Referring to the available microphotographs, it seems that our sizer is sensitive to scale 3 (spheres) in the first stage of the precipitation process then only to scale 4 (large agglomerates) in the second part.

As mentioned in section 2.4.2, spectral turbidity is monitored in main reactor. Experimental and theoretical aspects as well as applications of this method to crystallization are extensively developed in [17-20]. Figure 14 shows the variation of turbidity with time at different wavelengths. Time zero is taken when temperature reaches $60^{\circ} \mathrm{C}$. Although the signal is relatively confused, valuable information can be drawn for these results: 
AIChE Journal, 2009, 55(10), 2553-2562, doi : 10.1002/aic.11870

* from the experimental methods used in this work, turbidimetry is the only one sensitive to precipitation practically from its beginning, because the signal immediately deviates from baseline;

* up to 35 minutes, turbidity depends on wavelength, what is characteristic of the presence of submicronic and micronic particles in the medium;

* beyond 35 minutes, turbidity becomes independent of wavelength; this means that fine particles are either absent or "invisible". This point will be discussed later.

\section{Discussion}

Previous experimental results lead to a new vision of the mechanism of formation of the $\mathrm{ZnS}$ multi-scale agglomerates.

\section{Nature of the different agglomeration scales}

The succession of different agglomeration scales is currently allotted to transitions between hydrodynamic regimes [10], each of them corresponding to a characteristic scale. This type of interpretation is quite acceptable in the case of three successive scales ( 2 transitions) however cannot be appropriate here for four successive scales. Two hydrodynamic scales only are indeed relevant:

* the Batchelor scale of about 200 nm which marks the limit between Brownian and laminar shear flow agglomeration in the eddies of the turbulent structure;

* the Kolmogorov micro-scale represents the size of the smallest of these eddies (20 $\mu \mathrm{m}$ for the stirring rate of $700 \mathrm{rpm})$. Mekki-Berrada [12] proved that the agglomerate fourth scale was proportional to the Kolmogorov scale.

Batchelor size intervention in the agglomeration dynamics is less clear.

We think that in fact although four size scales are visible in the pictures, three only are in fact really concerned with agglomeration. Most convincing piece of evidence is the absence of scale 2 agglomerates ("caterpillars") which certainly constitute intrinsic morphological structures of the scale 3 spherical agglomerates. Agglomeration in fact proceeds according to two steps:

i) crystallites (scale 1) $\rightarrow$ sphere (scale 3)

ii) spheres $\rightarrow$ large agglomerate

Given final size of the spheres ( 1 to $3 \mu \mathrm{m}$ ) and of the crystallites ( $40 \mathrm{~nm}$ ) on the one hand, and Batchelor scale value $(200 \mathrm{~nm})$ on the other hand, crystallite agglomeration follows Brownian (perikinetic) dynamics and sphere agglomeration laminar (orthokinetic) dynamics [21].

Structure 2 onset certainly implies aspects more chemical than hydrodynamic. We think more particularly of oriented agglomeration and crystal growth.

\section{Dynamics of the different processes}

Figure 12 shows that the size of the spherical particles grows linearly with time whereas Williams et al. [7] found parabolic variation. From Figure 8, on the other hand, mass of precipitated $\mathrm{ZnS}$ appears also as a linear function of time. From these two types of behavior, one can deduce that the number of spherical particles itself varies as $t^{-2}$; this is an intermediary situation between Brownian and shear-flow agglomeration whereas the size range in question concerns this latter mechanism rather. This unusual behavior expresses certainly the fact that growth and agglomeration are certainly responsible jointly for the size of the agglomerates and their structuring. 
AIChE Journal, 2009, 55(10), 2553-2562, doi : 10.1002/aic.11870

\section{Mechanism of the homogeneous precipitation of zinc sulfide}

From previous results and observations we propose the following mechanism for the precipitation of zinc sulfite in the form of four-scale agglomerates;

* as turbidimetry shows, precipitation probably starts as soon as TAA decomposes ( $\mathrm{T}=$ $60^{\circ} \mathrm{C}$, instant zero of the process) ; as the final temperature level is reached shortly after, the rest of the process is considered as isothermal;

* then, during 30 to 45 minutes, a burst of nuclei is observed which results very rapidly in the formation of crytallites, both detected by turbidimetry (Figure 14) and observed in the SEM pictures (Figure 11); formation of spheres by agglomeration starts also and is visible by laser diffraction (Figure 13) and in the pictures;

* beyond 35 minutes, we mentioned that the isolated crystallites were no longer detected by turbidimetry whereas they certainly continue to be produced by nucleation, more particularly because the supersaturation level is high. Figures 11 and 12 tend to prove that they are integrated by the spherical particles, probably so quickly that they are not "seen" by the optical sensor. This inspires us a mechanism of secondary nucleation close to the surface of the spheres immediately followed by the integration of the new crystallites through a process of oriented agglomeration which could create the scale 2 structure;

* this phenomenon of growth of the spherical particles through direct integration of new crystallites apparently lasts throughout the experiment as proved by the continuous increase of third scale size which is observed on the microphotographs and reported in Figure 12; at the same time, the corresponding kernel of agglomeration of the spheres by shear-flow mechanism rapidly increases (proportional to the cube of the diameter) and the large agglomerates (scale 4) appear and develop up to the Kolmogorov size;

* we also should explain why the volume fraction in large agglomerate seems to leveloff after 110 minutes (Figure 13) whereas crystallites are still produced and feed the spherical particles; our explanation is relative to the properties of Fraunhoffer light diffraction which is essentially due to the outline of the objects (the large agglomerates) and is little influenced by possible finer internal changes (at the level of the spherical particles).

This approach of the mechanism of formation of $\mathrm{ZnS}$ leads us to contest the assumption suggested by [8-9] according to whom the formation of crystallite fibers (scale 2) would be done before the onset of the spherical agglomerates

\section{Conclusions}

The understanding of the mechanism of precipitation of the multi-scale agglomerates is of great importance to control their morphology and the associated properties.

From their nature, the agglomerates are often relatively fragile and their withdrawal is liable to introduce errors, whatever the interest of direct observations. This justifies the use of in situ, physicochemical and optical measurements which provide continuous information particularly adapted to the monitoring of complex systems.

Agglomerates with four successive scales are always observed in the case of the homogeneous precipitation of zinc sulfide. Hydrodynamics plays a crucial role to determine the size of the largest agglomerates; however, other factors should be invoked to interpret the complete structure.

The possibility of calculating in real-time the supersaturation level and the quantity of formed zinc sulfide as well as on line measurement of turbidity proved essential to understand the nature and the succession of the construction stages of the agglomerates. In addition to this breakthrough, consistency between experimental results of different nature 
AIChE Journal, 2009, 55(10), 2553-2562, doi : 10.1002/aic.11870

was a validation for the experimental methods themselves and for the different models used in particular kinetic.

The whole of the experimental results confirms that the mechanism utilizing independent agglomerations is widely questionable. We think rather that crystallites resulting from nucleation-growth (scale 1) agglomerate to produce the first spherical particles (scale 3). Then new crystallites seem to be produced near these spherical particles and stick directly to them. In the course of time, the spherical agglomerates seem to be structured and let appear the structure which we call scale 2. When the spherical particles are sufficiently large, they undergo shear-flow agglomeration which gives birth to scale 4 .

This work, although more quantitative than the precedent ones on multi-scale agglomeration deserves to be supplemented by an approach in term of population balance which would combine the various supposed stages and would allow to definitely their coherence.

\section{REFERENCES}

[1] Bowersox DF, Smith DM, Swift EH. The precipitation of zinc sulfide from acid solutions by thioacetamide. Talanta 1960:3: 282-295.

[2] Celikkaya A, Akinc M. Morphology of zinc sulfide particles produced from various zinc salts by homogeneous precipitation. J. Am. Ceram. Soc. 1990:73:245-250.

[3] Celikkaya A, Akinc M. Preparation and mechanism of formation of sphericalsubmicrometer zinc sulfide powders. J. Am. Ceram. Soc. 1990:73:23602365 .

[4] Chiu G. The preparation of monodisperse zinc sulfide sols. J. Coll. Inter. Sci. 1981:309-310.

[5] Vacassy R, Schloz SM, Dutta J, Pulmmer CJG, Houriet R, Hofmann H. Synthesis of controlled spherical zinc sulfide particles by precipitation from homogeneous solutions. J. Am. Ceram Soc. 1998:81:2699-2705.

[6] Wilhelmy DM, Matijevic E. Preparation and properties of monodispersed sphericalcolloidal particles of zinc sulfide. J. Chem. Soc.1984:80:563-570.

[7] Willams R, Yocom PN, Stofko FS. Preparation and properties of spherical zinc sulfide particles. J. Coll. Inter. Sci. 1985:106:388-398.

[8] Eshuis A, Koning CAJ. The mechanism of particle formation during homogenous precipitation of zinc sulfide. Colloid and Polymer Science. $1994: 272: 1240-1244$.

[9] Eshuis A, Van Elderen GRA, Koning CAJ. Colloid and Surfaces, Physicochemical and Engineering Aspects. 1999:151:505-512.

[10] David R, Espitalier F, Cameirao A, Rouleau L. Developments in the understanding and modeling of the agglomeration of suspended crystals in crystallization from solution. KONA Powder and Particle. 2003: 21:40-53.

[11] Cameirão A. Experimental study and modeling of a precipitation combined with agglomeration between crystals of different morphologies: case of strontium molybdate, Ph. D. dissertation, INP Toulouse, France, 2007.

[12] Mekki-Berrada MK. Synthèse d'agglomérats multi-échelles de sulfure de zinc par précipitation homogène, Ph. D. dissertation, Ecole des Mines de Saint-Etienne. France, 2007.

[13] Butler E, Peters DG, Swift EH. Hydrolysis reaction of thioacetamide in aqueous solutions. Analytical Chemistry. 1958:30:1379-1383.

[14] Peeters OM, de Ranter DJ. Pathways in thioacetamide hydrolysis in aqueous acid: Detection by kinetic analysis. J. Chem. Soc., Perkin 2. 1974:1832-1835, doi: 10.1039/P29740001832

[15] Sugimoto T. Reaction in homogeneous solutions of metal ions. In Fine Particles: Synthesis, Characterization, and Mechanisms of Growth, vol. 92 of Surfactant Science Series. Marcel Dekker New York. 2000:190-198.

[16] Crawley GM, Cournil M, Di Benedetto D. Size analysis of fine particle suspensions by spectral turbidimetry: potential and limits. Powder Technology. 1997:91:197-208. 
AIChE Journal, 2009, 55(10), 2553-2562, doi : 10.1002/aic.11870

[17] Sessiecq P, Gruy F, Cournil M. J. Cryst. Growth. Study of aluminium chloride crystallization in a mixed vessel 2000:208:555-568.

[18] Tontrup C, Gruy F, Cournil M. Turbulent aggregation of titania in water. J. Coll. Inter. Sci. 2000:229:511-525.

[19] Cournil M, Herri JM. Asymptotic model for gas liquid crystallization in two-film layers systems. AIChE. J. 2003:49:2030-2038

[20] Cournil M, Gruy F, Cugniet P. Influence of nonwetting on the aggregation dynamics of micronic solid particles in a turbulent medium, J. Coll. Inter. Sci. 2005: 284:54859 .

[21] De Boer GBJ, Hoedemakers GFM, Thoenes D Coagulation in turbulent flow Chem. Engng. Res. Des.1989:67:301-315.

[22] West CJ, Dorsey NE International Critical Tables of Numerical Data Physics, Chemistry and Technology, volume VI. McGRAW-HILL Book Company, first edition, 1929.

[23] Stokes RH, Robinson RA Electrolyte solutions. Butterworths scientific publications, London, $2^{\text {nd }}$ edition, 1959.

APPENDIX A: DETERMINATION OF TOTAL SULFUR CONCENTRATION IN THE AUXILIARY REACTOR FROM MEASUREMENTS OF THE ELECTRICAL CONDUCTIVITY

In the $\mathrm{pH}$ conditions of the auxiliary reactor $\left(\mathrm{pH}=13.5 ;\left[\mathrm{H}_{2} \mathrm{~S}\right] \approx 0\right)$, the total quantity of absorbed $\mathrm{H}_{2} \mathrm{~S},\left[S_{\mathrm{T}}\right]_{\mathrm{R} 2}$ is given by the mass balance equation :

$\left[S_{\mathrm{T}}\right]_{\mathrm{R} 2}=\left[\mathrm{HS}^{-}\right]+\left[\mathrm{S}^{2-}\right]$

Neglecting the $\mathrm{H}_{3} \mathrm{O}^{+}$ions concentration, the electroneutrality condition then reduces to:

$\left[\mathrm{OH}^{-}\right]_{\mathrm{to}}=\left[\mathrm{Na}^{+}\right]=\left[\mathrm{OH}^{-}\right]_{\mathrm{t}}+[\mathrm{HS}-]+2\left[\mathrm{~S}^{2-}\right]$

The acidity constant $K_{\mathrm{a} 2}$ and the autoionisation constant $K_{\mathrm{e}}$ are respectively given by:

$$
K_{a 2}=\gamma_{e q} \frac{\left[\mathrm{S}^{2-}\right]\left[\mathrm{H}^{+}\right]}{\left[\mathrm{HS}^{-}\right]} \quad K_{e}=\gamma_{e q}{ }^{2}\left[\mathrm{OH}^{-}\right]\left[\mathrm{H}_{3} \mathrm{O}^{+}\right]
$$

$\gamma_{\text {eq }}$, the average activity coefficient is equal to 0.73 for a concentration of $0.2 \mathrm{M}$ in $\mathrm{NaOH}$ at $\mathrm{T}=25{ }^{\circ} \mathrm{C}$ [22]. We consider too that the basic solution of the auxiliary reactor is little affected by the $\mathrm{H}_{2} \mathrm{~S}$ absorption.

Hence the relation between $\left[S_{\mathrm{T}}\right]_{\mathrm{R}_{2}}$ and $\left[\mathrm{S}^{2-}\right]$

$$
\left[\mathrm{S}^{2-}\right]^{2}-\left[\mathrm{S}^{2-}\right]\left(C_{0}+\frac{K_{e}}{K_{a 2} \gamma_{e q}}\right)+\left[\mathrm{S}_{\mathrm{T}}\right]_{R 2}\left(C_{0}-\left[\mathrm{S}_{\mathrm{T}}\right]_{R 2}\right)=0
$$

with: $C_{\mathrm{o}}=\left[\mathrm{OH}^{-}\right]_{\mathrm{to}}$

The electrical conductivity in the auxiliary reactor is itself linked to the different ionic concentrations $c_{\mathrm{i}}$ by the relation:

$$
\sigma_{\text {sol }}=\sum_{i} c_{i} \lambda_{i}
$$

Where $\lambda_{\mathrm{i}}$ is the equivalent ionic conductivity of species $i$. Thus :

$$
\sigma_{\text {sol }}=\left[\mathrm{Na}^{+}\right] \lambda_{\mathrm{Na}^{+}}+\left[\mathrm{OH}^{-}\right] \lambda_{\mathrm{OH}^{-}}+\left[\mathrm{HS}^{-}\right] \lambda_{\mathrm{HS}^{-}}+\left[\mathrm{S}^{2-}\right] \lambda_{\mathrm{S}^{2-}}
$$

Using now the Kohlrausch additivity law [23]: $\lambda_{\mathrm{AB}}=\lambda_{\mathrm{A}}+\lambda_{\mathrm{B}}$, we obtain:

$$
\sigma_{\text {sol }}(t)=\left[\mathrm{Na}^{+}\right] \lambda_{\mathrm{NaOH}}+\left[\mathrm{HS}^{-}\right]\left(\lambda_{\mathrm{NaHS}}-\lambda_{\mathrm{NaOH}}\right)+2\left[\mathrm{~S}^{2-}\right]\left(\lambda_{\mathrm{Na}_{2} \mathrm{~S}}-\lambda_{\mathrm{NaOH}}\right)
$$


AIChE Journal, 2009, 55(10), 2553-2562, doi : 10.1002/aic.11870

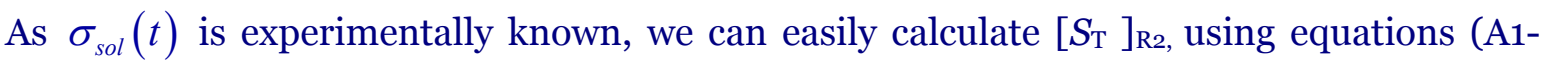
A3).

This calculation method is particularly robust, because little dependent on $K_{\mathrm{a} 2}$ value which is moreover relatively badly known.

APPENDIX B: DETERMINATION OF THE TOTAL SULFUR CONCENTRATION IN THE MAIN REACTOR FROM KINETIC CALCULATION

As explained in main text, we use the Peeters and de Ranter model [14] (presented earlier in the "Chemical aspects" section) to describe the TAA decomposition mechanism.

The different reaction rates allow us to express the variation of the concentrations with time:

$$
\begin{array}{lll}
\frac{\mathrm{d}\left[\mathrm{H}_{2} \mathrm{~S}\right]}{\mathrm{d} t}=k_{1} C_{1}+k_{4} C_{3} & \frac{\mathrm{d} C_{1}}{\mathrm{~d} t}=-\left(k_{1}+k_{2}\right) C_{1} & \frac{\mathrm{d} C_{3}}{\mathrm{~d} t}=k_{2} C_{1}-k_{4} C_{3} \\
\frac{\mathrm{d}\left[\mathrm{NH}_{3}\right]}{\mathrm{d} t}=k_{3} C_{2}+k_{2} C_{1} & \frac{\mathrm{d} C_{2}}{\mathrm{~d} t}=k_{1} C_{1}-k_{3} C_{2} & \frac{\mathrm{d} C_{4}}{\mathrm{~d} t}=k_{3} C_{2}+k_{4} C_{3}
\end{array}
$$

with $C_{1}=[\mathrm{TAA}], C_{2}=\left[\mathrm{CH}_{3} \mathrm{CONH}_{2}\right], C_{3}=\left[\mathrm{CH}_{3} \mathrm{CSOH}\right]$ and $C_{4}=\left[\mathrm{CH}_{3} \mathrm{COOH}\right]$.

The values of reaction rates $k_{1}$ to $k_{4}$ can be found in [14] for different temperature values.

At initial instant $t=\mathrm{o}, C_{2}, C_{3}$ and $C_{4}$ are zero. Assuming that the temperature remains constant throughout the decomposition, analytical integration of the previous differential equations is possible and gives in particular:

$$
\frac{\mathrm{d}\left[\mathrm{H}_{2} \mathrm{~S}\right]}{\mathrm{d} t}=\frac{C_{0}}{k_{4}-k_{1}-k_{2}}\left[\left(k_{1}+k_{2}\right)\left(k_{4}-k_{1}\right) \mathrm{e}^{-\left(k_{1}+k_{2}\right) t}-k_{2} k_{4} \mathrm{e}^{-k_{4} t}\right]
$$

Hence the possibility of calculating $\left[\mathrm{H}_{2} \mathrm{~S}\right](t)$ and the total sulfur concentration because at the $\mathrm{pH}$ values prevailing in the main reactor: $\left[S_{\mathrm{T}}\right]_{\mathrm{R} 1}=\left[\mathrm{H}_{2} \mathrm{~S}\right]$. 


\section{Figures}

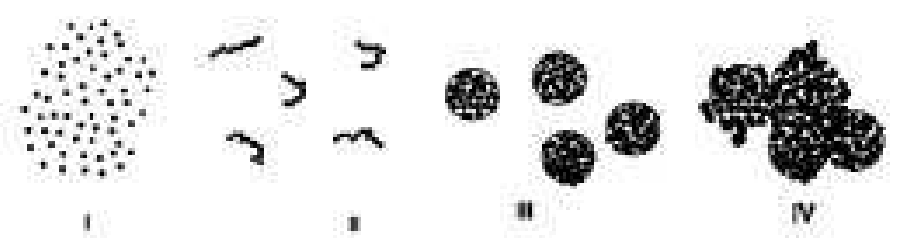

Figure 1: Schematic representation of the steps of multiscale agglomeration according to Refs 8 and 9

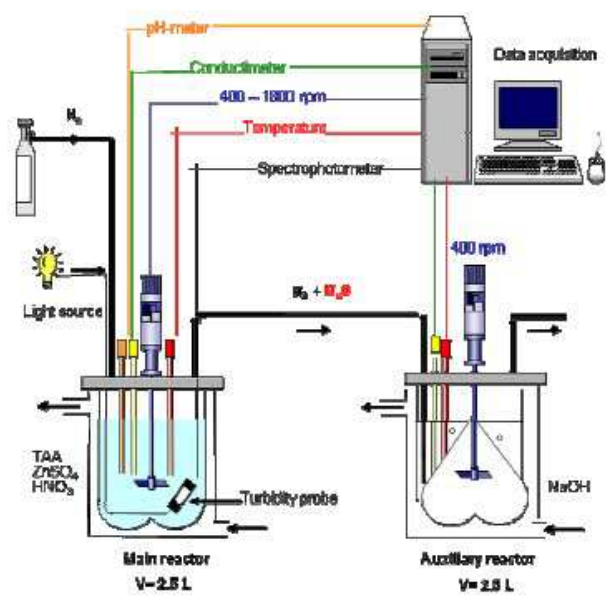

Figure 2: Experimental Set-Up

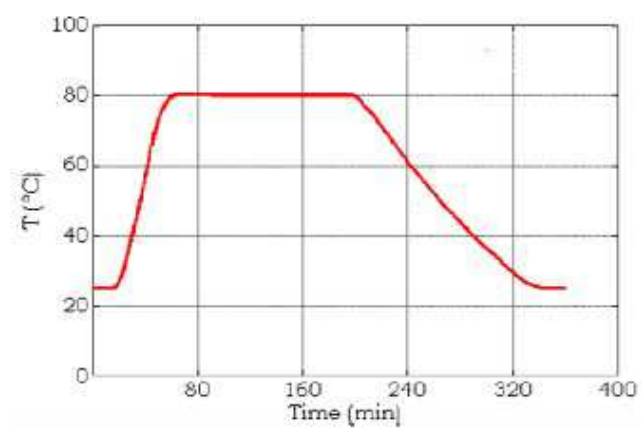

Figure 3: Temperature programming in main reactor

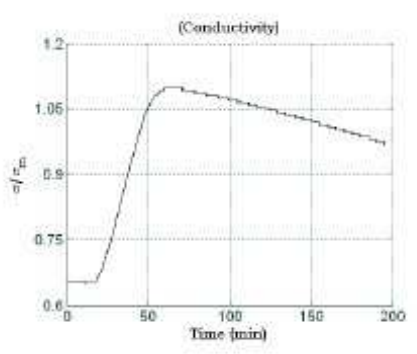

(a)

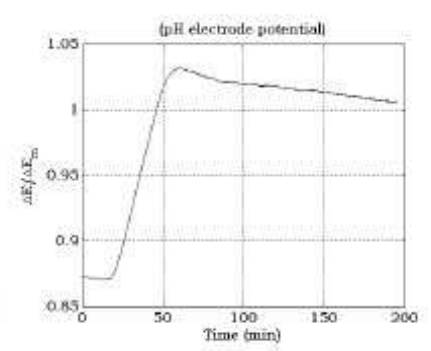

(b)

Figure 4: Electrical conductivity (a) and $\mathrm{pH}$ electrode potential (b) during the $\mathrm{ZnS}$ precipitation process in the main reactor 
AIChE Journal, 2009, 55(10), 2553-2562, doi : 10.1002/aic.11870

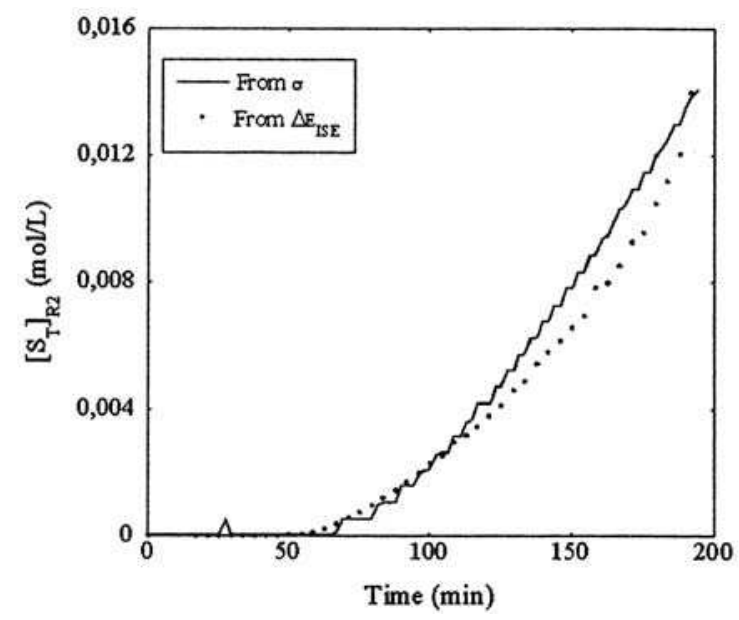

Figure 5: Variation of total sulfur concentration with time in the auxiliary reactor

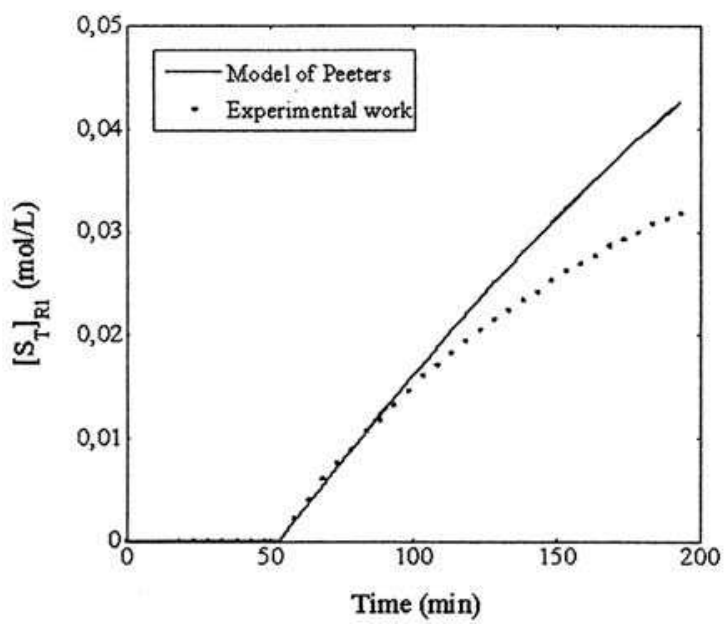

Figure 6: Total sulfur concentration versus time in main reactor (according to [14]:solid line; our experimental work: dotted line)

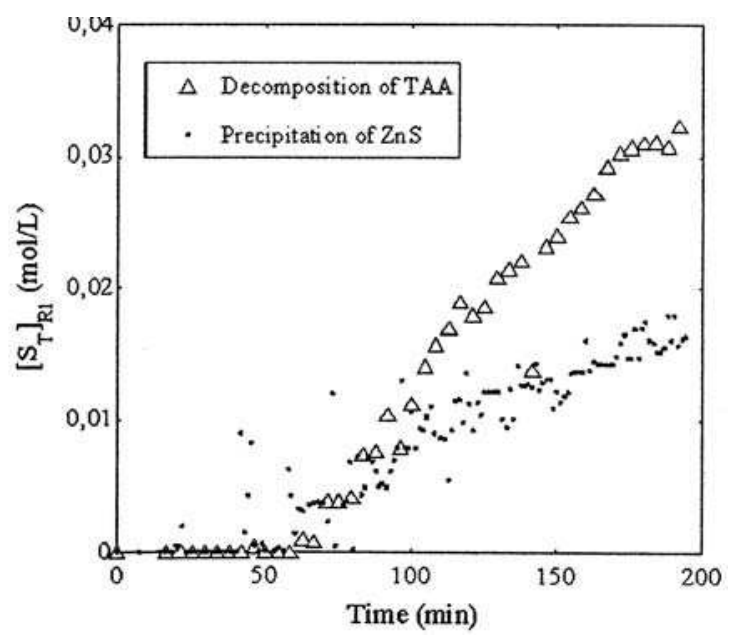

Figure 7: Total sulfur concentration versus time in the experimental system (in absence and in presence of zinc ions) 
AIChE Journal, 2009, 55(10), 2553-2562, doi : 10.1002/aic.11870

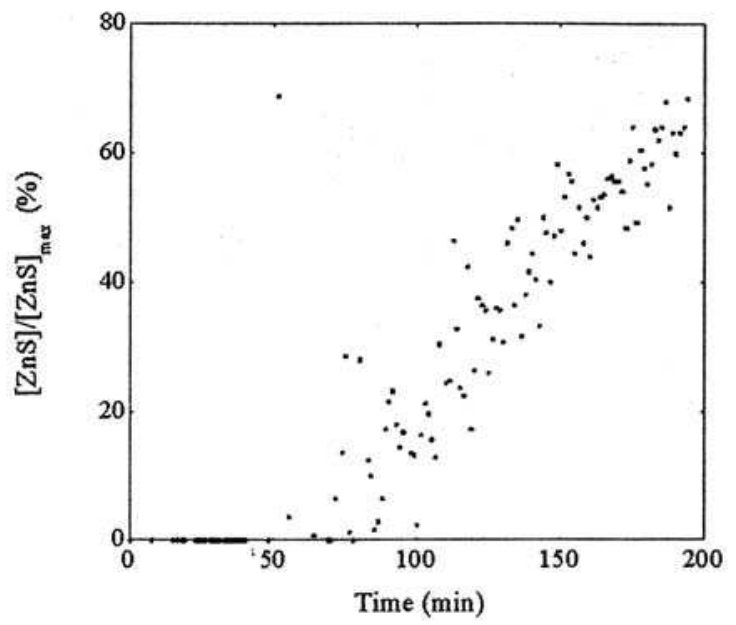

Figure 8: Precipitation yield versus time in main reactor

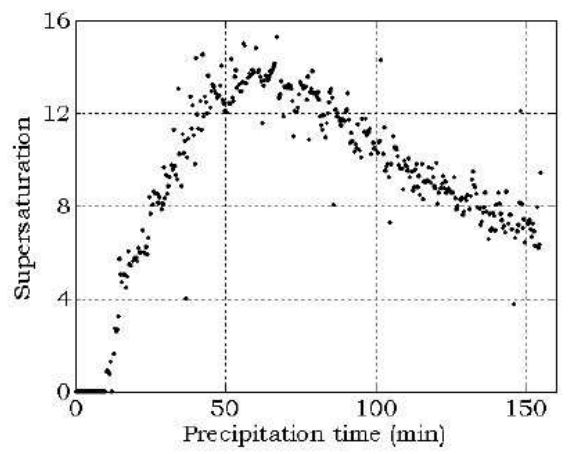

Figure 9: variation of supersaturation level in main reactor 
AIChE Journal, 2009, 55(10), 2553-2562, doi : 10.1002/aic.11870

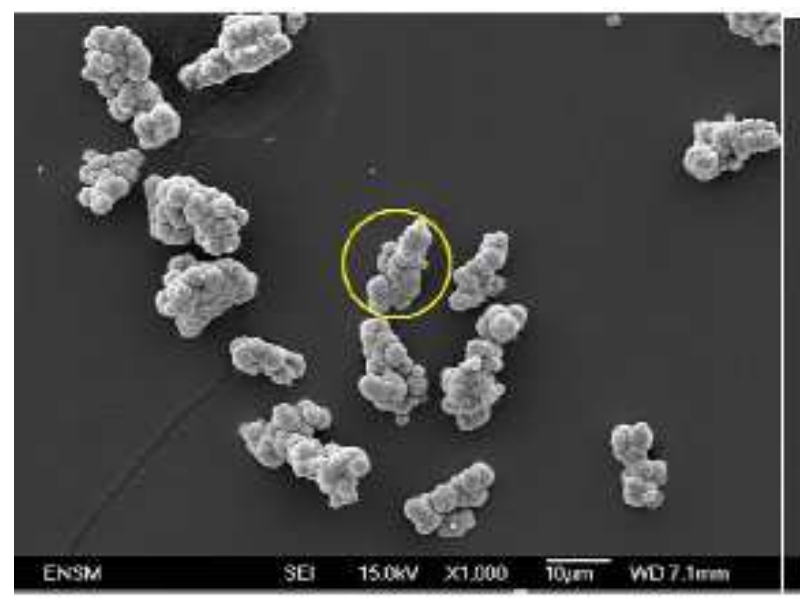

(a) General view



(b) Fourth scale

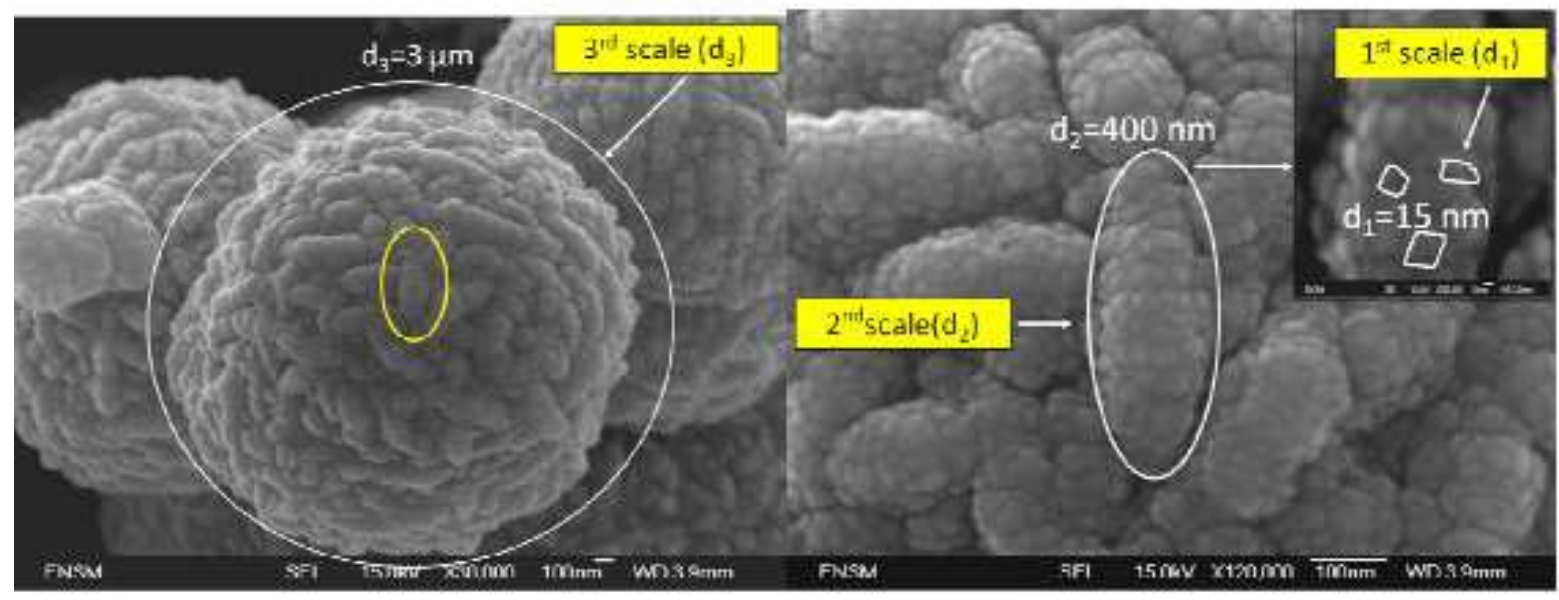

c) Third scale

(d) Second and first scales

Figure 10: ZnS agglomerate morphology (samples removed at the end of the experiment) 
AIChE Journal, 2009, 55(10), 2553-2562, doi : 10.1002/aic.11870
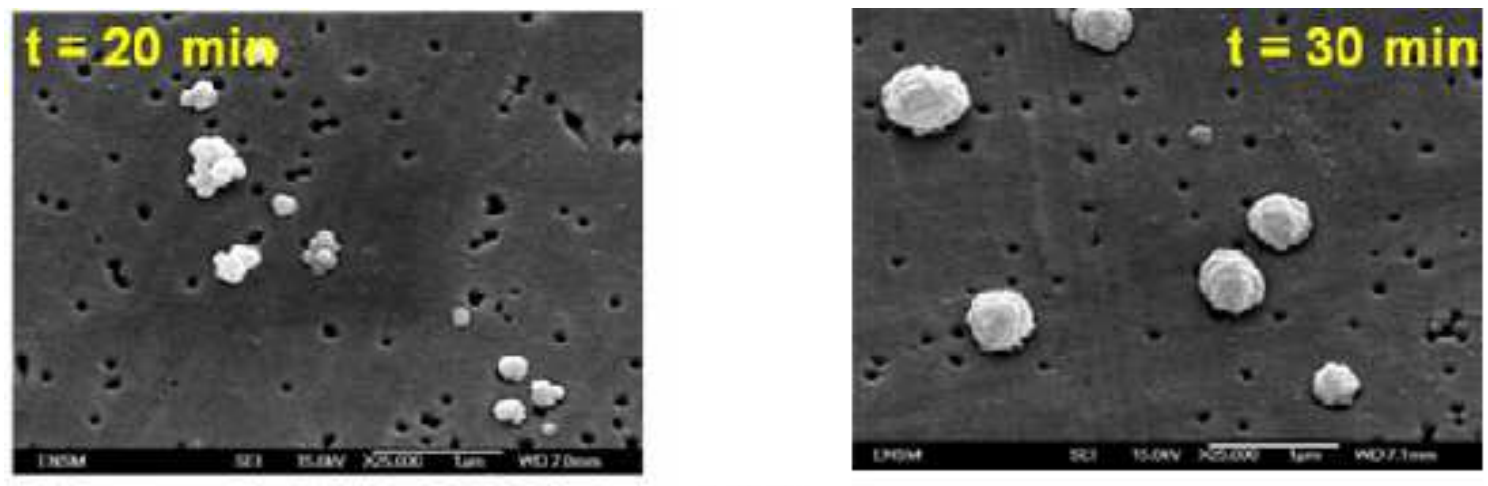

$1 \mu \mathrm{m}$
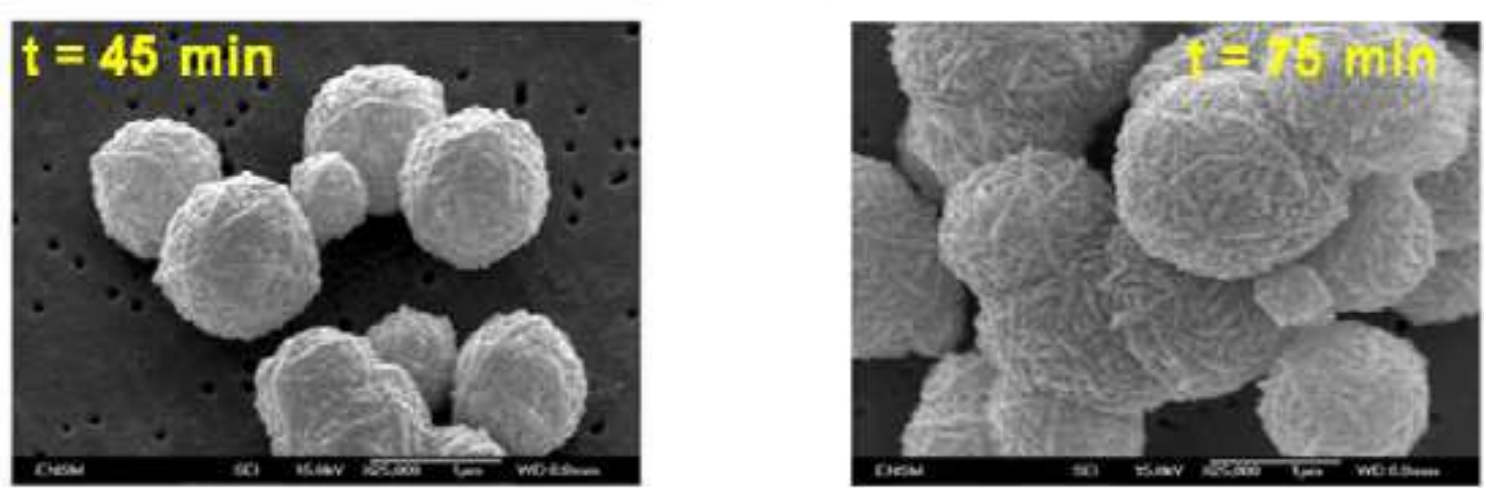

Figure 11: Morphology of $\mathrm{ZnS}$ samples withdrawn at different precipitation times

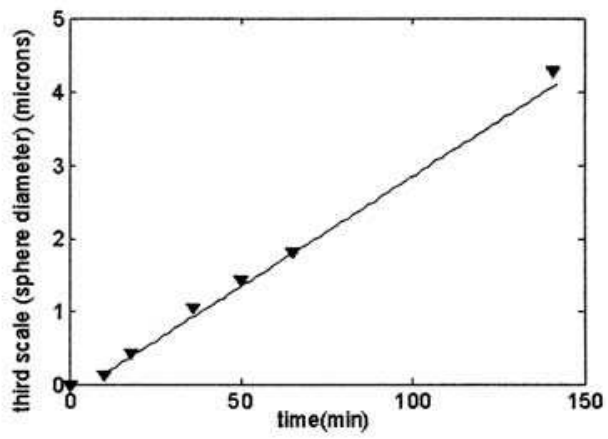

Figure 12. Variation versus time of the agglomerate third scale size (from SEM microphotographs)

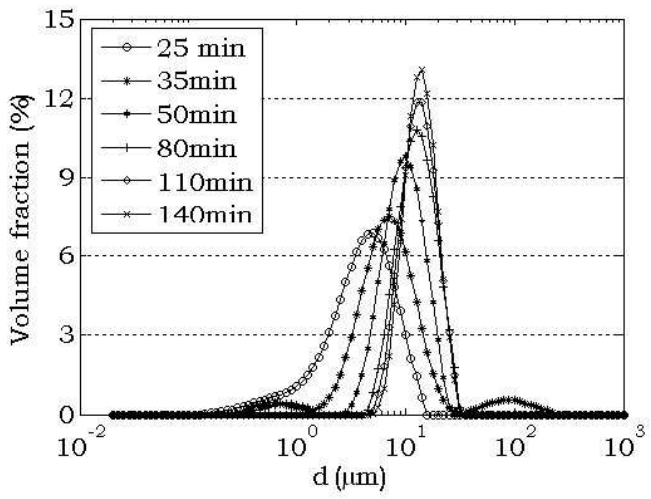

Figure 13. Particle size distribution at different precipitation times (Malvern 200o) 
AIChE Journal, 2009, 55(10), 2553-2562, doi : 10.1002/aic.11870

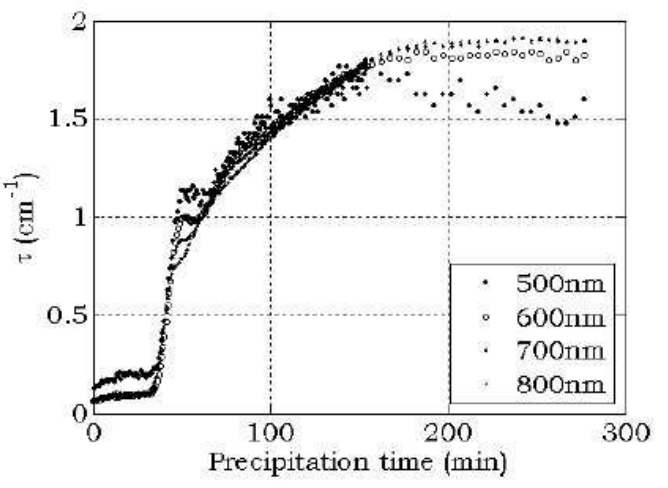

Figure 14. Variation of turbidity with time throughout the precipitation 